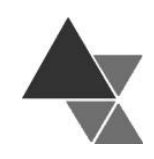

\title{
Iniquidades e promoção pública de ambientes alimentares saudáveis na cidade de Curitiba, Brasil
}

\author{
Natália Ferreira de Paula1, Nilson Maciel de Paula² e Roberto Eduardo Bueno ${ }^{3}$
}

O objetivo deste artigo é analisar a distribuição espacial dos equipamentos de disponibilidade de frutas e hortaliças $(\mathrm{FH})$ no município de Curitiba à luz do princípio da equidade, tendo por base pesquisa ecológica nas 10 regionais do município. Para tanto foram considerados o perfil econômico, a densidade de equipamentos públicos que comercializam FH e a correlação desses indicadores com o estado nutricional de adultos residentes na área estudada. Os resultados das análises revelam que as regionais mais pobres apresentaram maior prevalência de adultos acima do peso e menor densidade de equipamentos públicos que comercializam $\mathrm{FH}$. Portanto, há uma negligência ao princípio da equidade, tendo em vista o comportamento mercadológico na distribuição dos equipamentos públicos beneficiando em maior proporção aqueles já privilegiados pelo sistema alimentar neoliberal.

Palavras-chave: Segurança Alimentar e Nutricional; Abastecimento de alimentos; Política nutricional; Estado nutricional; Meio ambiente e saúde pública.

\section{Inequality and public promotion of healthy food environments in the city of Curitiba, Brazil}

The aim of this paper is to analyze the geographic distribution of supplying equipment of fruits and vegetables (FV) in Curitiba, state of Paraná, Brazil, following the principle of equity, through an ecological research carried out in ten urban regions. For this purpose, aspects such as economic profile, spatial density of state equipment, namely stalls and boxes, trading FV and their relationship with nutritional local State of adult residents, were taken into account. The main findings of this analysis have shown a higher prevalence of overweight adults twined with lower density of state equipment selling FV in the poorer areas. Therefore, as detected in this investigation, the principle of equity embedded in the guidelines of National Policy of Food Security and Nutrition has not been complied with. Local State equipment has been in line with market requirements, which tend to benefit those already profiting from the neoliberal food system, while poorer segments living in the fringes of urban network remain exposed to the odds of supply-demand relations in order to access healthy food.

Keywords: Food security and nutrition; Food supply; Nutrition policy; Nutritional status; Environment and public health.

1 Docente do curso de graduação em nutrição da UFPR, do Unicuritiba e da UniCesumar. Doutoranda do Programa de Pós-Graduação em Políticas Públicas da UFPR. E-mail: nataliafesr13@gmail.com. ID ORCID: https://orcid.org/0000-0002-5205-2369. Endereço para correspondência: Rua Martim Afonso no 257 apto. 32, São Francisco - Curitiba - Paraná. Tel.: (41) $9.9218-1548$

2 Professor sênior do Programa de Pós-Graduação em Políticas Públicas da Universidade Federal do Paraná. ID ORCID: https://orcid.org/0000-0002-2143-5172.

3 Professor do Programa de Pós-Graduação em Políticas Públicas da UFPR. ID ORCID: https://orcid.org/0000-0001-5546-8397 


\section{INTRODUÇÃO}

A dinâmica do Sistema Alimentar atual é responsável por uma série de desdobramentos globais e locais, entre eles o que se tem denominado de Sindemia Global, este termo caracteriza a sinergia entre eventos pandêmicos de obesidade, desnutrição e mudanças climáticas ${ }^{[1]}$. Este panorama tem se intensificado pela crise causada pela pandemia de COVID-19, a qual tem contribuído para o aprofundamento das desigualdades sociais e violações do Direito Humano à Alimentação Adequada ${ }^{[2]}$.

Desta forma, ressalta-se aqui a necessidade de relacionar os determinantes do processo sindêmico apresentado acima, com a correlação de forças que movimentam o Estado para um lado ou para outro, promovendo ou violando os direitos sociais. Nesse sentido, destaca-se a avaliação da comissão The Lancet apontando a necessidade de enfrentar os sistemas políticos, socioeconômicos e comerciais, como aspectos intrínsecos ao problema da obesidade, indicando medidas entre as quais está a promoção de ambientes alimentares mais saudáveis ${ }^{[1]}$.

Os ambientes alimentares contemplam os espaços de aquisição e consumo de alimentos, como locais de estudo, trabalho e religião, além do espaço residencial da população, o qual será explorado nesse estudo, e que por sua vez, reflete as condições socioeconômicas das pessoas que ali residem, resultando assim numa segregação espacial. Tendo por base essa segmentação espacial como um traço comum das cidades brasileiras, observa-se que áreas de maior renda e maior escolaridade contam com um número maior e mais diverso de estabelecimentos que comercializam alimentos in natura[ ${ }^{[3]}$. Neste sentido, o processo de ocupação demográfica das áreas urbanas, as ações do capital no mercado imobiliário, os fluxos migratórios, mesmo que sujeito a ações regulatórias de governos locais, acaba determinando a exposição desigual da população aos ambientes alimentares. Contudo, essa iniquidade impacta o estado nutricional da população, pesquisas demonstram que tanto a desnutrição como a obesidade e as doenças crônicas não transmissíveis atingem mais agressivamente as pessoas mais vulneráveis economicamente ${ }^{[4,5]}$. A exemplo do que ocorre no Brasil, onde as pessoas com menores graus de escolaridade são as que possuem maiores índices de doenças crônicas não transmissíveis causadas pela má alimentação, como diabetes e hipertensão ${ }^{[6]}$. Portanto equipamentos públicos que comercializam frutas e hortaliças $(\mathrm{FH})$ podem contribuir para reduzir essa desigualdade na disponibilidade de alimentos saudáveis em locais onde residem pessoas com menor renda. Nesse sentido, as ações de Segurança Alimentar e Nutricional (SAN) na malha urbana devem ser norteadas pelo princípio da equidade, como recomendado pela Política Nacional de Segurança Alimentar e Nutricional (PNSAN) [7].

Diante do exposto, este artigo, pautado pelo princípio da equidade, tem como objetivo analisar a distribuição espacial intra-urbana dos equipamentos públicos de disponibilidade de FH no município de Curitiba e avaliar o grau de correlação entre o estado nutricional e a renda da população. Esta inquietação surgiu a partir da constatação da atual pandemia de sobrepeso e obesidade causada por inúmeros fatores, entre eles o aumento do consumo de alimentos ultraprocessados e a redução do consumo de alimentos in natura, como $\mathrm{FH}^{[1,8,9]}$. Padrão de consumo que tem sido influenciado pela dinâmica dos ambientes alimentares ${ }^{[10]}$, os quais compõem as estruturas de abastecimento alimentar.

\section{METODOLOGIA}

O estudo utiliza como metodologia a pesquisa ecológica, a qual "compara a ocorrência da doença/condição relacionada à saúde e à exposição de interesse entre agregados de indivíduos para 
verificar a possível existência de associação entre elas" [11]. Dessa forma, a condição relacionada a saúde é aqui ilustrada pelo estado nutricional da população, no qual os indivíduos são agregados a partir das regionais administrativas do município e a exposição é dada tanto pela condição socioeconômica, como pelo número de equipamentos públicos de comercialização de $\mathrm{FH}$ no espaço residencial.

\section{Características socioeconômicas do município}

O município de Curitiba, situado a 934 metros acima do nível do mar, possui aproximadamente 43.503 hectares (ha), distribuídos em 75 bairros, que estão divididos em 10 regionais administrativas, onde residem aproximadamente 1.879.355 habitantes com rendimento médio mensal de $\mathrm{R} \$ 3.497,70{ }^{[12]}$. Seu Índice de Desenvolvimento Humano (IDH) de 0,823 é o 4o maior entre as capitais brasileiras ${ }^{[12]}$ e seu Coeficiente de Gini é de 0,55[13]. Além disso, há uma dimensão espacial da desigualdade social, a qual pode ser percebida em Curitiba quando se compara por exemplo a renda das pessoas que residem no bairro Caximba (bairro periférico) que é de $\mathrm{R} \$ 1.613,24$ às pessoas que moram no bairro do Batel (bairro central) $\mathrm{R} \$ 9.821,57$, demonstrando que a desigualdade não só está presente, como também pode ser percebida pela segregação espacial da população ${ }^{[11]}$.

\section{Amostragem e indicadores}

Tendo por base a pesquisa ecológica, adotou-se como unidades de análise as 10 regionais do município (Figura 1), cujos aspectos considerados são o perfil econômico da população, a densidade de equipamentos públicos que comercializam FH (para verificar a relação da frequência de equipamentos com a população da regional) e a correlação desses indicadores com o estado nutricional de adultos residentes na área geográfica.
Figura 1. Mapa das administrações regionais do município de Curitiba

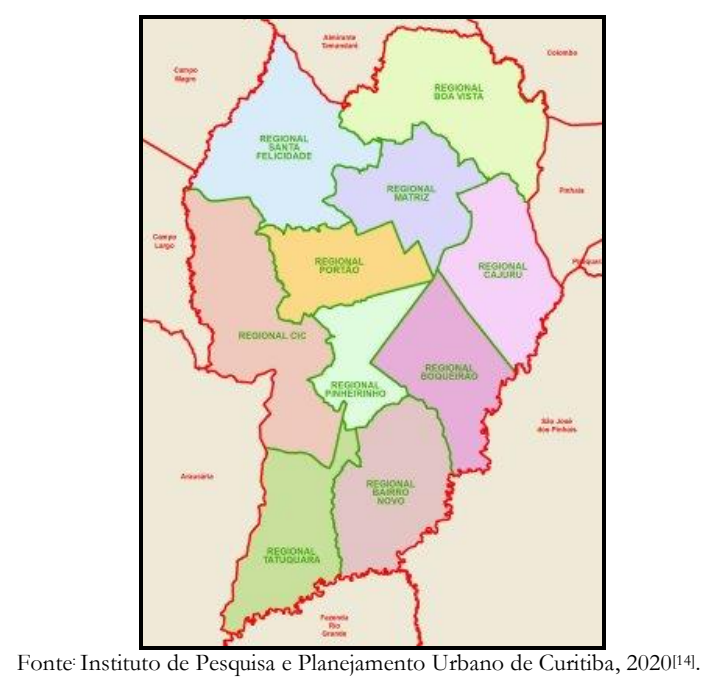

As fontes dos dados, bem como a fórmula de cálculo para indicadores estão listadas no Quadro 1. Os equipamentos contemplados são Sacolão da Família, Programa Direto da Roça e Mar, Feiras Livres, Feiras Orgânicas, Programa Nossa Feira, Mercado Municipal, Mercado regional e Mercado de Orgânicos. Sendo equipamentos que oferecem alimentos prontos para o consumo (como Restaurante Popular) ou realizam doação de alimentos (Banco de Alimentos), não foram contabilizados, pois não atendem os requisitos de inclusão para a pesquisa (comercialização de FH). 
Quadro 1. Procedimento utilizado para obtenção dos indicadores

\begin{tabular}{|c|c|c|}
\hline Indicador & Fórmula de cálculo & $\begin{array}{c}\text { Fonte dos } \\
\text { dados }\end{array}$ \\
\hline $\begin{array}{l}\text { Densidade de } \\
\text { equipamentos } \\
\text { públicos que } \\
\text { comercializam } \\
\text { frutas e hortaliças } \\
(\mathrm{FH})^{*}\end{array}$ & $\begin{array}{l}\text { Número de } \\
\text { equipamentos na } \\
\text { regional x } 1.000 \\
\text { População total da } \\
\text { regional }\end{array}$ & $\begin{array}{l}\text { Site da SMAB } \\
\text { IPPUC** } \\
\text { Estimativa } \\
\text { para } 2016\end{array}$ \\
\hline $\begin{array}{l}\text { Proporção }(\%) \text { de } \\
\text { adultos acima do } \\
\text { peso }\end{array}$ & $\begin{array}{l}\text { Proporção }(\%) \text { de } \\
\text { adultos em estado de } \\
\text { sobrepeso na regional } \\
+\% \text { de adultos em } \\
\text { estado de obesidade na } \\
\text { regional }\end{array}$ & $\begin{array}{l}\text { SISVAN*** } \\
\text { Municipal } \\
\text { Dados de } 2018\end{array}$ \\
\hline $\begin{array}{l}\text { Rendimento } \\
\text { mensal médio }\end{array}$ & $\begin{array}{l}\text { Rendimento mensal } \\
\text { médio por regional }\end{array}$ & $\begin{array}{l}\text { IPPUC** } \\
\text { Relatório } \\
\text { Nosso Bairro } \\
2015\end{array}$ \\
\hline
\end{tabular}

Notas: *Fórmula adaptada de Castro Júnior, 2018[15].

*Dado obtido no site do Instituto de Pesquisa e Planejamento Urbano de Curitiba (IPPUC). **Dado obtido por meio do Sistema de Vigilância Alimentar e Nutricional/Secretaria Municipal de Saúde de Curitiba.

Fonte: Elaborado pelos autores (2019).

Os dados relativos à quantidade e localização dos equipamentos foram coletados no site da Secretaria Municipal do Abastecimento (SMAB) em março de 2019 e em seguida classificados por regional a partir do endereço disponibilizado no site.

\section{Estratificação de renda}

Para o tratamento dos dados de renda foi realizada a classificação das regionais em renda alta, renda média e renda baixa: 1 . regionais com renda alta: Matriz, Portão, Santa Felicidade e Boa Vista, com rendimento mensal médio de $\mathrm{R} \$ 6.438$, $\mathrm{R} \$ 5.297, \mathrm{R} \$ 4.823$ e $\mathrm{R} \$ 3.727$, respectivamente; 2. regionais com renda média: Cajuru ( $\mathrm{R} \$ 3.314)$, Boqueirão ( $\mathrm{R} \$ 2.837)$ e Pinheirinho ( $\mathrm{R} \$ 2.888) ; 3$. regionais com renda baixa: CIC (R $\$ 2.148)$, Bairro Novo (R\$2.012) e Tatuquara ( $\mathrm{R} \$ 1.674)$.

\section{Estratificação do estado nutricional}

Os dados relativos ao estado nutricional também foram divididos em duas faixas, reunindo as regionais abaixo da média (que é de $70 \%$ ) de adultos acima do peso e aquelas acima da média de adultos acima do peso.

\section{Análise estatística}

Após a obtenção dos indicadores foi realizada a correlação entre os mesmos por meio do Software Microsoft Excel, com o qual foi também calculado o coeficiente de correlação de Pearson (R) e a significância estatística (valor de $p$ ).

Os dados também foram incorporados ao sistema Q-GIS que gerou mapa contendo intensidade de densidade de equipamentos por regional, rendimento mensal médio e adultos acima do peso (Figura 2).

\section{Programas de disponibilidade de frutas e hortaliças na cidade de Curitiba}

A cidade de Curitiba é considerada referência mundial na implementação e gestão de programas de SAN, entre as ações de comercialização de FH estão as feiras convencionais e orgânicas, sacolão da família, mercado municipal, mercado de orgânicos, mercado regional e programa direto da roça e mar. Essas ações têm contribuído significativamente para o Sistema Municipal de SAN do município.

As feiras orgânicas e convencionais comercializam $\mathrm{FH}$, além de outros alimentos em diversos pontos da cidade, sendo algumas vezes comercializados pelos(as) próprios(as) agricultores(as) familiares. O programa Nossa Feira é uma tenda móvel, instalada em ponto predefinido permanecendo no local por 4 horas, onde são comercializados FH a R $\$ 2,29$, diretamente pelos(as) produtores(as) da Região Metropolitana de Curitiba. O Sacolão da Família também comercializa FH a $\mathrm{R} \$ 2,29$, mas possui pontos fixos e horário comercial de funcionamento. $O$ mercado municipal e o mercado regional são compostos por lojas e boxes, onde comerciantes vendem grande variedade de alimentos, entre eles, FH. Anexo ao mercado municipal, na região central, encontra-se um espaço determinado para comercialização de alimentos orgânicos e agroecológicos, denominado Mercado de Orgânicos de Curitiba. O Programa Direto da Roça e Mar é constituído por tendas móveis instaladas em determinados pontos da cidade durante um dia específico, comercializando $\mathrm{FH}$, 
pescados e frutos do mar, oriundos do litoral do Paraná e de agricultores da região.

Observa-se que os equipamentos de comercialização de FH são numerosos e diversificados, possuindo potencial para promover ambientes alimentares que estimulam estilos de vida saudáveis (oásis alimentares). Contudo, é preciso analisar como essas ações estão distribuídas na cidade, e quem são seus principais beneficiários. Vários estudos sobre o planejamento urbano de Curitiba demonstraram o caráter espacial da segregação social, a qual se elevou nas últimas cinco décadas, quando os investimentos públicos negligenciaram os espaços marginalizados, agravando ainda mais a desigualdade social. Contudo, este processo foi camuflado pelas estratégias de marketing da cidade divulgando amplamente uma imagem de cidade modelo[16,17], contendo informações enaltecedoras também no campo da SAN.

Paralelamente, o debate sobre a evolução do terceiro regime alimentar aponta entre outros fatores, para as desigualdades no estado nutricional, na dieta alimentar saudável e também nos ambientes alimentares saudáveis, o que permite relacioná-las com a dinâmica do neoliberalismo. Desta forma, este estudo visa identificar se as ações de SAN implementadas em Curitiba atendem o princípio da equidade, ou se seguem a lógica do regime neoliberal e aprofundam o modelo excludente produzido pelo planejamento urbano da cidade modelo. Desta forma, nos próximos tópicos são apresentados os resultados e discussões desta análise.

\section{RESULTADOS}

Os resultados expressam a correlação entre a condição socioeconômica da população, a distribuição espacial dos equipamentos públicos de comercialização de FH e o estado nutricional dos curitibanos e curitibanas.

Os equipamentos públicos de distribuição de FH totalizaram 88, sendo que a quantidade de equipamentos por regional variou de 1 (regional Tatuquara) à 28 (regional Matriz), conforme apresentado na Tabela 1.

Tabela 1. Distribuição dos equipamentos de comercialização de FH nas regionais adminstrativas de Curitiba

\begin{tabular}{|c|c|c|c|c|c|c|c|}
\hline Regional & $\begin{array}{l}\text { Sacolão da } \\
\text { Família }\end{array}$ & $\begin{array}{c}\text { Feira } \\
\text { convencional }\end{array}$ & $\begin{array}{c}\text { Feira } \\
\text { orgânica }\end{array}$ & $\begin{array}{l}\text { Mercado } \\
\text { municipal }\end{array}$ & Nossa Feira & Roça e Mar & $\begin{array}{l}\text { Total por } \\
\text { Regional }\end{array}$ \\
\hline Bairro novo & 2 & 0 & 0 & 0 & 0 & 0 & 2 \\
\hline Boa vista & 3 & 5 & 0 & 0 & 3 & 3 & 14 \\
\hline Boqueirão & 3 & 2 & 0 & 0 & 0 & 0 & 5 \\
\hline Matriz & 1 & 12 & 12 & 1 & 1 & 1 & 28 \\
\hline Cidade Industrial & 1 & 0 & 0 & 0 & 1 & 0 & 2 \\
\hline Cajuru & 1 & 3 & 0 & 1 & 2 & 0 & 7 \\
\hline Pinheirinho & 1 & 0 & 0 & 0 & 1 & 0 & 2 \\
\hline Portão & 2 & 7 & 4 & 0 & 0 & 0 & 13 \\
\hline Santa Felicidade & 1 & 7 & 1 & 0 & 2 & 3 & 14 \\
\hline Tatuquara & 1 & 0 & 0 & 0 & 0 & 0 & 1 \\
\hline Total Curitiba & 16 & 36 & 18 & 2 & 10 & 7 & 88 \\
\hline
\end{tabular}

Fonte: Elaborado pelos autores (2019)

A regional da Matriz conta com seis tipos diferentes de equipamento, enquanto as regionais
Bairro Novo e Tatuquara possuem apenas um. Nas regionais de maior renda média destacam-se as 
Feiras convencionais, Feiras orgânicas e Programa direto da roça e mar. Este último, por exemplo, possui sete pontos de comercialização, todos concentrados nas regionais da Matriz, Santa Felicidade e Boa Vista. Assim como as Feiras orgânicas que também estão localizadas estritamente nas regionais mais estruturadas, como Matriz, Portão e Santa Felicidade.

As Feiras convencionais contam com 36 pontos na cidade, que por sua vez estão situados em seis das 10 regionais. Contudo apresentam-se em maior quantidade nas três regionais de maior renda média, enquanto estão ausentes nas quatro regionais de maior vulnerabilidade econômica. O Programa Nossa Feira possui dez pontos de comercialização, localizados em seis regionais, sendo elas Boa Vista, Santa Felicidade, Cajuru, Matriz, CIC e Pinheirinho. Ou seja, o Programa que segundo a prefeitura foi criado para atender os bairros mais pobres $\mathrm{e}$ afastados do Centro, não está atendendo as Regionais com menor renda mensal média, que são Tatuquara e Bairro Novo. Já o Sacolão da Família por sua vez, possui 16 unidades e é o único equipamento presente em todas as regionais.

No que diz respeito à distribuição espacial dos dados antropométricos de adultos acima do peso, Curitiba apresenta maior prevalência de sobrepeso e obesidade em pessoas pobres como demonstrado pela correlação entre perfil econômico da regional e adultos acima do peso (Gráfico $1 \mathrm{e}$ Figura 2). Observa-se assim uma forte correlação negativa entre as variáveis, indicando que quanto menor nível socioeconômico da população da regional, maior a prevalência de adultos acima do peso.

Soma-se a esse resultado a disparidade da densidade de equipamentos entre as regionais. Os resultados do estudo demonstram que nas regiões mais pobres existe uma menor densidade de equipamentos públicos que comercializam $\mathrm{FH}$, conforme retratado no gráfico 2, onde foi detectada forte correlação negativa entre a densidade de equipamentos por 1.000 habitantes por regional e adultos acima do peso.

Gráfico 1. Correlação entre perfil econômico das regionais de Curitiba e adultos acima do peso

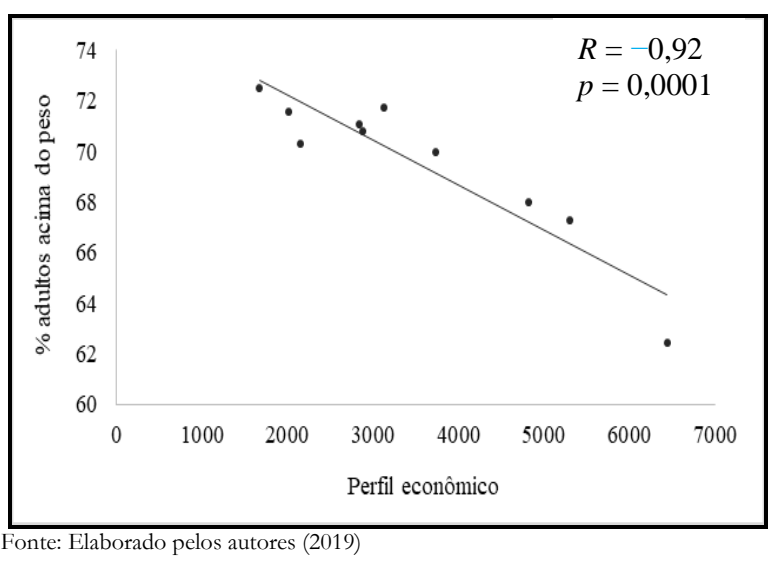

Gráfico 2. Correlação entre densidade de equipamentos por 1.000 habitantes por regional e adultos acima do peso

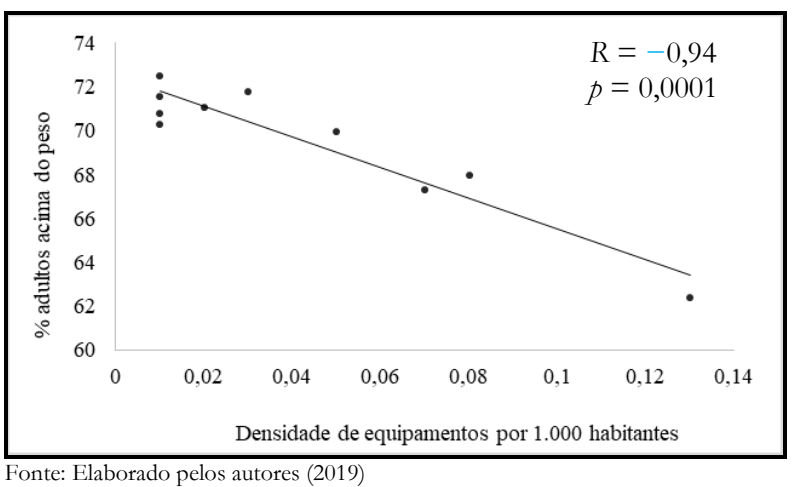

Em seguida é exibido o Mapa (Figura 2) que retrata as evidências apresentados nas tabelas e gráficos. As regionais CIC, Tatuquara e Bairro Novo, coloridas em vermelho escuro são as que possuem menor renda familiar média, menor densidade de equipamentos por 1.000 habitantes, e uma proporção acima de $70 \%$ dos adultos em obesidade e sobrepeso. 
Figura 2. Mapa das regionais de Curitiba e sua correlação com o estado nutricional, renda e densidade de equipamentos por 1.000 habitantes

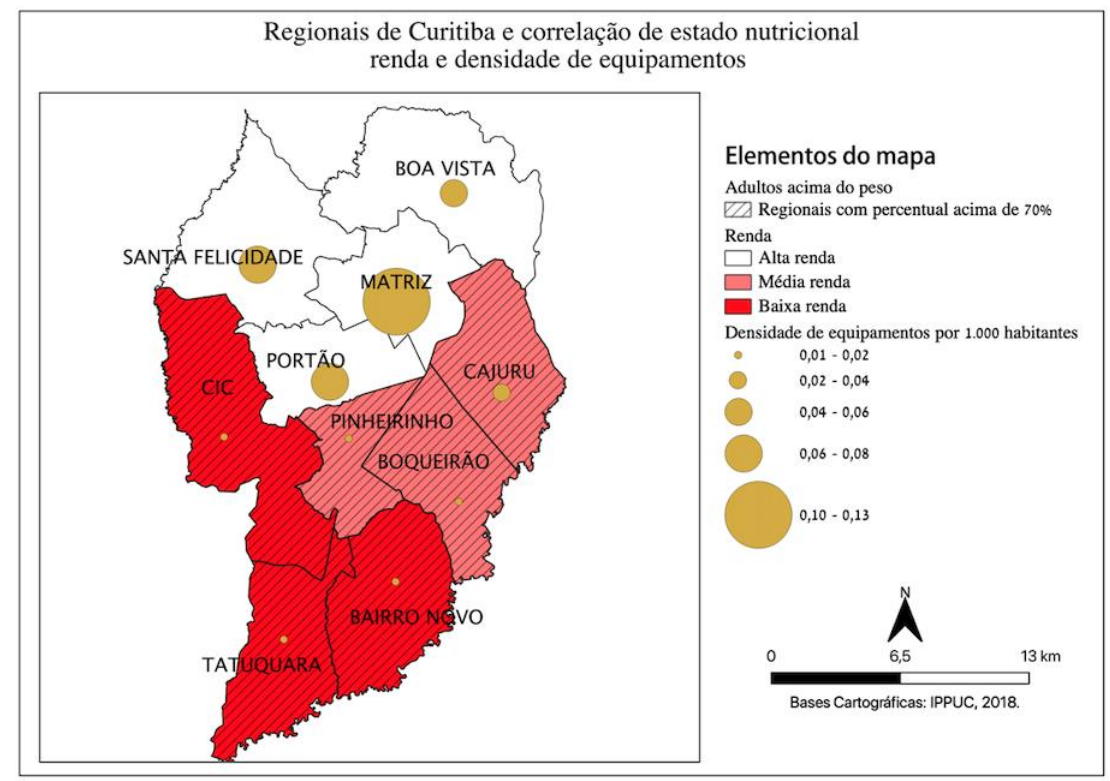

Fonte: Elaborado pelos autores (2019)

Em contraste estão as regionais Boa Vista, Matriz, Santa Felicidade e Portão, que possuem maior renda familiar média, maior densidade de equipamentos por 1.000 habitantes e percentual abaixo de $70 \%$ de adultos acima do peso. Já as regionais Cajuru, Boqueirão, Pinheirinho e Bairro Novo possuem dados intermediários aos dois extratos extremos de renda e estado nutricional das regionais apresentados anteriormente.

\section{DISCUSSÃO}

Os resultados apresentados anteriormente evidenciam uma maior quantidade e diversidade de equipamentos públicos de disponibilidade de $\mathrm{FH}$ nas áreas de melhores níveis socioeconômicos da cidade. Esses achados dialogam com os pensamentos de Boyd Swinburn et al ${ }^{4]}$, Gerardo Otero et al[5] e Julie Guthman ${ }^{[18]}$, para os(as) quais o atual regime alimentar é fortemente marcado por diferenças de classe. Julie Guthman ${ }^{[18]}$ destaca ainda que o acesso a alimentos específicos está relacionado à classe social, raça e gênero. De acordo com a autora algumas razões para explicar essas diferenças são variáveis como custo dos alimentos, educação e acesso. A educação tem sido fator importante para demonstrar ainda, profundas disparidades de estado nutricional na população brasileira. No ano de $2018,86,3 \%$ da população de 0 a 8 anos de escolaridade estavam em estado de sobrepeso ou obesidade, enquanto entre aqueles(as) com escolaridade de 12 anos ou mais esse percentual era de 67,1, o que corresponde a uma diferença de aproximadamente 20 pontos percentuais ${ }^{[6]}$. Esses dados corroboram com os achados de Marion Devaux et a ${ }^{19]}$ em pesquisa realizada na Austrália, Canadá, Inglaterra e Coreia, os(as) quais observaram que as pessoas com menor tempo de estudo apresentaram maiores índices de obesidade.

A disparidade espacial referente à densidade de equipamentos de comercialização de $\mathrm{FH}$ pode ser um fator potencializador do resultado do estado nutricional da população. Em estudo de correlação entre ambiente alimentar, ambiente para prática de atividade física e índice de massa corporal (IMC), Paulo Cesar de Castro Júnior ${ }^{[15]}$ encontrou associação inversa entre IMC de mulheres e ambientes, indicando que quanto melhor $\mathrm{O}$ ambiente alimentar e de atividade física menor o IMC, ou seja, quanto mais opções para comprar alimentos saudáveis no local de moradia, menor a prevalência de sobrepeso e obesidade em mulheres brasileiras. Milene Pessoa et al20] também comprovaram maior consumo de FH nas zonas de 
níveis de renda mais altos. Mariana Fernandes Fortes et al[3] identificaram que a falta de estabelecimentos que comercializam alimentos in natura e minimamente processados em bairros periféricos, faz com que a população apresente dificuldades em seguir as recomendações do Guia Alimentar para a População Brasileira[21], as quais são baseadas em alimentos in natura, como FH.

Essa é portanto um questão que reflete no consumo alimentar, que já é desproporcional no Brasil. Tendo por base a Pesquisa de Orçamento Familiar (POF) de 2017-2018, observa-se que a aquisição domiciliar per capita anual de frutas em domićlios brasileiros, apresenta uma diferença muito expressiva, chegando a $313 \%$ mais para domicílios com classes de rendimento total e variação patrimonial mensal familiar de $\mathrm{R} \$ 14.310$ em comparação aos domicílios com classes de rendimento total e variação patrimonial mensal familiar de $\mathrm{R} \$ 1.908^{[22]}$. Esta disparidade também é demonstrada para aquisição domiciliar per capita anual de hortaliças, a qual chega a uma diferença de $187 \%$ quando comparadas as mesmas faixas extremas de rendimento total e variação patrimonial mensal familiar [22]. A Pesquisa Nacional de Saúde (PNS) de 2019 mostra que o consumo recomendado de FH para adultos no Brasil reflete os dados de aquisição apresentados pela POF 20172018. Em Curitiba a proporção de pessoas com rendimento de até $1 / 4$ do salário mínimo que consomem FH no mínimo cinco vezes na semana é de $5 \%[23]$. Em contrapartida a proporção de pessoas com rendimento que supera cinco salários mínimos representa $31,9 \%$, ou seja, a proporção de pessoas com melhores condições socioeconômicas que consomem FH conforme o recomendado, representa mais que o sêxtuplo da população mais pobre que consegue atingir esse padrão alimentar[23].

Esses dados da POF e da PNS retratam o maior acesso a FH para as classes mais altas, reforçando a importância do papel dos equipamentos públicos de comercialização de $\mathrm{FH}$ para atendimento da população de pessoas com menores níveis socioeconômicos. Dessa forma, a menor disponibilidade de equipamentos públicos de distribuição de $\mathrm{FH}$ encontrada nos bairros mais pobres de Curitiba pode contribuir para o menor consumo desses alimentos, visto que esses equipamentos oferecem, além de maior disponibilidade dos alimentos, melhor preço (alguns apresentam preço único), melhor procedência (grande parte é direto do produtor familiar), alimentos orgânicos e agroecológicos (mais saudáveis), atributos essenciais para a promoção da SAN e para a redução dos impactos de "ambientes obesogênicos" que propiciam o aumento da obesidade ${ }^{[4,24]}$.

Os ambientes obesogênicos, em sua essência, são reflexos da centralidade das grandes corporações no sistema alimentar e de decisões políticas e econômicas de governos que afetam as estruturas de produção e consumo de alimentos ${ }^{[5]}$. Embora intervenções da iniciativa privada, como formulação de produtos com ingredientes mais saudáveis (orgânicos) devam ser consideradas, a restrição dos ambientes obsesogênicos será mais efetiva com ações do Estado por meio da priorização de práticas agroecológicas, proibição de alimentos não saudáveis, guias alimentares, equipamentos públicos de alimentação e nutrição, entre outros. Ainda de acordo com Boyd Swinburn et $a^{\left.l^{4}\right]}$, políticas públicas que modifiquem ambientes alimentares têm um impacto mais abrangente se comparadas com programas de educação e promoção da saúde, visto que afetam toda a população, são sustentáveis, tornam-se sistêmicas e modificam comportamentos.

Os ambientes alimentares podem ser classificados como desertos alimentares quando possuem o acesso a alimentos saudáveis limitado, decorrente da baixa disponibilidade de estabelecimentos ${ }^{[25]}$. De acordo com os resultados desse estudo, que teve como objetivo pesquisar apenas o "ambiente alimentar público", se pode indicar que em relação a distribuição de equipamentos públicos de disponibilidade de $\mathrm{FH}$ as áreas mais pobres da cidade são desertos alimentares públicos. Portanto, essa análise identifica uma violação ao princípio da equidade presente na PNSAN, por parte da intervenção pública de alocação de equipamentos de disponibilidade de FH no município analisado.

O princípio de equidade em saúde passou a ser debatido a partir de 1986 pela Organização Mundial da Saúde (OMS), sendo contemplada na Carta de Ottawa, documento oriundo da Primeira Conferência Internacional sobre Promoção da 
Saúde ${ }^{[26]}$. No Brasil, esse princípio foi incorporado ao Sistema Único de Saúde (SUS) [27], a Lei Orgânica de Segurança Alimentar e Nutricional (LOSAN), além de outros marcos institucionais do país[28]. Para John Rawls ${ }^{[2]}$, frente à iniquidade como uma expressão da desigualdade injusta, pode-se considerar tratamento desigual justo, quando é benéfico ao indivíduo mais carente. Ou seja, tendo em vista que iniquidades são desigualdades criadas por um processo de transformações produtivas e de concentração de renda e riqueza ao longo da história do capitalismo, os indivíduos mais vulneráveis devem receber tratamento diferente por meio de mecanismos de proteção acionados pelo Estado.

Esta avaliação é relevante quando se observa a proeminência da orientação neoliberal na condução das políticas governamentais no Brasil no período atual, as quais têm reduzido e até descartado o papel do Estado como rede de proteção ao empobrecimento de grande parte da população e ao aprofundamento das desigualdades socioeconômicas. A preservação do status quo tem sido amparada pela concentração de poder dos segmentos mais afluentes da sociedade, pelas estratégias de grandes corporações e pela própria lógica do sistema financeiro ${ }^{[30]}$.

Tendo por base a política econômica mundial hegemônica moldada pelos princípios neoliberais, Gerardo Otero et al[5] analisaram o consumo de alimentos e renda, cujos resultados permitiram a formulação do conceito de "dieta neoliberal". Foi assim identificado, um padrão de consumo de alimentos desigual, de forma que os grupos de renda mais alta têm maior acesso a alimentos de qualidade, como frutas, hortaliças, carnes e vinhos importados, enquanto nos segmentos sociais mais vulneráveis é maior a ingestão de calorias vazias, açúcares, sal, óleos e gorduras, com óbvio impacto em seu estado nutricional. Nesse sentido, observa-se que "as escolhas alimentares estão estruturalmente condicionadas pela desigualdade de renda"[5](p.2). A noção de dieta neoliberal, na realidade, repercute a dinâmica do sistema alimentar neoliberal. Ou seja, "comemos o que enormes produtores e distribuidores de alimentos oligopolistas tem para oferecer, que por sua vez é moldado ou facilitado pela intervenção do Estado neoliberal"[5]. Estas são, portanto, dimensões parciais da ordem neoliberal do capitalismo contemporâneo, contagiando todos os aspectos da sociedade.

Paralelamente à dieta neoliberal, existe uma manifestação da noção de regime alimentar neoliberal, adotada por Gerardo Otero et al[5], como desdobramento da formulação original de regime alimentar de Harriet Friedmann e Philip McMichael[31]. Nesse sentido, o regime alimentar neoliberal é caracterizado pela liberalização do comércio e outras estruturas reguladoras estatais e supra-estatais que favorecem grandes corporações, como os processadores e varejistas de alimentos, além de oligopólios de insumos agrícolas e o mercado financeiro, cuja lucratividade é alavancada pela proliferação da dieta neoliberal[5].

Previamente, Andrea Freeman[32] observou fenômeno semelhante, o qual denominou "opressão alimentar", que se configura por um conjunto de fatores que expõe desigualmente as comunidades afro-americanas e latinas dos Estados Unidos ao consumo de fast-food em detrimento aos alimentos saudáveis. Entre esses fatores estão o marketing direcionado, a infiltração de valores falsamente modernizantes nas escolas, os subsídios do governo, o guia alimentar e a proliferação de estabelecimentos que comercializam alimentos industrializados em bairros pobres naquele país ${ }^{[32]}$.

A transição para a dieta neoliberal é um fenômeno global com fortes manifestações nos países em desenvolvimento, onde se propaga rapidamente ${ }^{[5]}$. Estudo publicado pela Organização Pan-Americana de Saúde demonstra que, tanto a venda de produtos ultraprocessados, quanto o aumento de peso e obesidade na América Latina têm aumentando num ritmo mais acelerado do que a média global[33]. Esse fato tem intima relação com os ambientes alimentares, e sobre esta questão Boyd Swinburn $\left.e t a{ }^{4}\right]$ afirma que "a obesidade é fruto de pessoas que respondem normalmente ao ambiente obesogênico a que estão expostas". Dessa forma, o papel do Estado, tanto por meio das decisões políticas, quanto pela disponibilidade equitativa de equipamentos de produção e abastecimento de alimentos saudáveis é crucial para promoção da SAN e efetivação do DHAA. 


\section{CONCLUSÃO}

Estas análises confirmam resultados obtidos por pesquisas que identificaram a segregação no processo de ocupação e construção do espaço urbano em Curitiba, que resultou na atual distribuição da cidade, a qual é dividida entre áreas ocupadas por pessoas de maior renda e aquelas ocupadas pelas de menor renda. Essa distribuição de renda está diretamente relacionada com o estado nutricional da população, demonstrando maior concentração de adultos acima do peso em áreas marginalizadas. Isso corrobora com a ideia de dieta neoliberal, na qual há uma combinação entre desigualdade social e um desigual padrão de consumo de alimentos saudáveis, assim como seu estado nutricional.

As diversas intervenções públicas para promover um ambiente alimentar saudável em Curitiba são potentes para reduzir os impactos dos ambientes obesogênicos e desertos alimentares. Entretanto, as ações analisadas não abarcam toda a cidade, são irregularmente distribuídas e privilegiam as regiões onde a renda é mais alta, ou seja, a segregação espacial permanece também nas intervenções de SAN do município. Os resultados desta análise revelam uma sintonia entre a distribuição de equipamentos públicos de comercialização de $\mathrm{FH}$ e o próprio planejamento urbano da cidade de Curitiba, os quais são igualmente excludentes e aprofundam as desigualdades sociais.

Os equipamentos públicos deveriam ter um papel no sentido contrário, a fim de minimizar essa discrepância na disponibilidade e acesso a alimentos saudáveis, que normalmente já é imposta e reproduzida pelo mercado. Dessa forma, a imagem de Curitiba como referência nas ações de SAN deve ser observada com cautela, assim como a cidade modelo, visto que privilégios são mantidos, num reflexo das tendências globais do capitalismo sob o manto do neoliberalismo. Justamente por esse motivo, talvez não seja efetivo transferir todos os equipamentos já existentes para as áreas de menor renda, visto que os mesmos foram formulados para a elite e muitos não se encaixariam na realidade, e principalmente na renda da população residente nas regiões mais pobres. Neste sentido, é necessário desenvolver outros equipamentos que atendam as especificidades dessa população e garantam a disponibilidade de FH de origem ecológica, a preço acessível, variedade e quantidades adequadas, atendendo o Direito Humano à Alimentação Adequada de todos(as) os(as) curitibanos(as).

Esses achados apontam ainda para a ocorrência de iniquidades na aplicação do orçamento público voltado para promoção da SAN, mais especificamente sobre a disponibilidade de alimentos saudáveis. Visto que para contemplar o princípio da equidade previsto na PNSAN, os equipamentos devem atender desigualmente em maior densidade as regiões mais pobres, que nesse caso, apresentaram também o estado nutricional da população mais comprometido. Neste sentido, o presente estudo visa prover subsídios para políticas públicas de SAN que reduza desigualdades sociais e beneficie os mais vulneráveis do ponto de vista nutricional.

\section{REFERÊNCIAS}

[1] The Lancet. Comissão sobre Obesidade. A Sindemia Global da Obesidade, Desnutrição e Mudanças Climáticas. Alimentando Políticas. Org. Instituto Brasileiro de Defesa do Consumidor (IDEC) [Internet.] 2019 [acesso em 2020 nov 20]. Disponível em: https://alimentandopoliticas.org.br/wpcontent/uploads/2019/08/idec-the_lancetsumario_executivo-baixa.pdf

[2] Daufenback V, Coelho DEP, Bógus CM. Sistemas alimentares e violações ao direito humano à alimentação adequada: reflexões sobre a pandemia de Covid-19 no Brasil. Segur. Aliment. Nutr. [Internet]. 2021 [acesso em 2021 mar 6]; 28:1-13. Disponível em: file:///Users/Michel/Downloads/8661745-

Texto\%20do\%20artigo-95112-1-10-20210304.pdf

[3] Fortes MF, Borges CA, Miranda WC, Jaime PC. Mapeando as desigualdades socioeconômicas na distribuição do comércio varejista local. Segur. Aliment. Nutr. [Internet]. 2018 [acesso em 2020 nov 20];25(3):4558. Disponível em: https://periodicos.sbu.unicamp.br/ojs/index.php/san/a rticle/view/8651966/18736

[4] Swinburn BA, Sacks G, Hall KD, McPherson K, Fine good DT, Moodie ML. The global obesity pandemic: shaped by global drivers and local environments. The Lancet [Internet]. 2011 [acesso em 2020 nov 20];378:804$814 . \quad$ Disponível em: 
https://www.thelancet.com/journals/lancet/article/PIIS 0140-6736(11)60813-1/fulltext

[5] Otero G, Pechlaner P, Gürcan EC. The Neoliberal Diet: Fattening Profit and People. The Routledge Handbook of Poverty in the United States. Edited Haymes SN; Haymes MV; Miller RJ. 2015.

[6] Brasil. Ministério da Saúde. Vigitel Brasil 2018 [Internet]. Brasília; 2019. [acesso em 2020 nov 20]. Disponível em: https://portalarquivos2.saude.gov.br/images/pdf/2019/ julho/25/vigitel-brasil-2018.pdf

[7] Brasil. Decreto no 7272, de 25 de agosto de 2010. Regulamenta a Lei no 11.346, de 15 de setembro de 2006, que cria o Sistema Nacional de Segurança Alimentar e Nutricional - SISAN com vistas a assegurar o direito humano à alimentação adequada, institui a Política Nacional de Segurança Alimentar e Nutricional PNSAN, estabelece os parâmetros para a elaboração do Plano Nacional de Segurança Alimentar e Nutricional, e dá outras providências. Diário Oficial da União. 2010 ago 28.

[8] Organização Panamericana de Saúde (OPAS) e Organização Mundial de Saúde (OMS). Alimentos y bebidas ultraprocesados en América Latina: tendencias, efecto sobre la obesidad e implicaciones para las políticas públicas [Internet]. Washington D.C: OPAS/OMS; 2015 [acesso em 2020 nov 20]. Disponível em: http://iris.paho.org/xmlui/bitstream/handle/123456789 /7698/9789275318645_esp.pdf

[9] Louzada ML. Consumption of ultra-processed foods and obesity in Brazilian adolescents and adults. Prev Med Dec [Internet]. 2015 [acesso em 2020 nov 20];81:9-15. Disponível em: https://www.ncbi.nlm.nih.gov/pubmed/26231112

[10] Ohri-Vachaspati P, Leviton LC. Measuring Food Environments: A Guide to Available Instruments. Am J Health Promot [Internet]. 2010 [acesso em 2020 nov 20];24(6):410-426. Disponível em: https://pubmed.ncbi.nlm.nih.gov/20594098

[11] Lima-Costa MF, Barreto SM. Tipos de estudos epidemiológicos: conceitos básicos e aplicações na área do envelhecimento. Epidemiologia e Serviços de Saúde [Internet]. 2003 [acesso em 2020 mai 06];12(4):189-201. Disponível http://scielo.iec.gov.br/pdf/ess/v12n4/v12n4a03.pdf

[12] Curitiba. Instituto de Pesquisa e Planejamento Urbano de Curitiba (IPPUC). Nossa Curitiba. Perfil
Físico-Territorial e Socioeconômico da Cidade de Curitiba; 2015.

[13] Atlas do Desenvolvimento Humano no Brasil. [Internet]. 2020 [acesso em 2020 nov 29]. Disponível em: http://www.atlasbrasil.org.br/consulta/planilha

[14] Curitiba. Instituto de Pesquisa e Planejamento Urbano de Curitiba. Mapa das administrações regionais do município de Curitiba. IPPUC [Internet]. 2020 [acesso em 2020 nov 29]. Disponível em: http://www.ippuc.org.br/mapas/351.html

[15] Castro Junior PCP. Ambiente alimentar comunitário medido e percebido: descrição e associação com Índice de Massa Corporal de adultos brasileiros [tese]. Rio de Janeiro: Fundação Oswaldo Cruz; 2018.

[16] Oliveira D. Curitiba e o mito da cidade modelo. Curitiba: Editora da UFPR; 2000.

[17] Carvalho AS, Sugai MI. A produção da cidade segregada: o caso de Curitiba. Segundo Simpósio de Estudos Urbanos: a dinâmica das cidades e a produção do espaço. Eixo temático: Produção do Espaço Urbano [Internet]. 2013 [acesso em 2020 nov 29]. Disponível em: http://www.fecilcam.br/anais/ii_seurb/documentos/car valho-andre-de-souza.pdf

[18] Guthman J. Weighing. In: Obesity, Food Justice, and the Limits of Capitalism. Berkeley: California University Press; 2011.

[19] Devaux M, Sassi F, Church J, Cecchini M, Borgonovi, F. Exploring the Relationship Between Education and Obesity. OECD Journal: Economic Studies [Internet]. 2011 [acesso em 2020 nov 29];1. Disponível em: http://dx.doi.org/10.1787/eco_studies2011-5kg5825v1k23

[20] Pessoa MC, Mendes LL, Caiaffa WT, Malta DC, Velásquez-Meléndez G. Availability of food stores and consumption of fruit, legumes and vegetables in a Brazilian urban area. Nutr Hosp [Internet]. 2015 [acesso em 2020 nov 29];31(3):1438-1443. Disponível em: https://pubmed.ncbi.nlm.nih.gov/25726245

[21] Brasil. Ministério da Saúde. Secretaria de Atenção à Saúde. Departamento de Atenção Básica. Guia alimentar para a população brasileira [Internet]. Brasília; 2014 [acesso em 2020 nov 29]. Disponível em: https://bvsms.saude.gov.br/bvs/publicacoes/guia_alime ntar_populacao_brasileira_2ed.pdf 
[22] Instituto Brasileiro de Geografia e Estatística IBGE. Pesquisa de Orçamentos Familiares 2017-2018: avaliação nutricional da disponibilidade domiciliar de alimentos no Brasil [Internet]. Rio de Janeiro; 2020 [acesso em 2020 nov 29]. Disponível em: https://biblioteca.ibge.gov.br/visualizacao/livros/liv101 704.pdf

[23] Instituto Brasileiro de Geografia e Estatística IBGE. Coordenação de Trabalho e Rendimento. Pesquisa Nacional de Saúde. Microdados. Informações sobre estilos de vida. Alimentação: Brasil, grandes regiões e unidades de federação. Rio de Janeiro; IBGE; 2019.

[24] Swinburn B, Egger G, Raza F. Dissecting obesogenic environments: the development and application of a framework for identifying and prioritizing environmental interventions for obesity. Prev Med. 1999;29:563-570.

[25] Beaulac J, Kristjansson E, Cummins S. A systematic review of food deserts, 1966-2007. Prev Chronic Dis. 2009;6(3):A105.

[26] First International Conference on Health Promotion, Ottawa, 21 November 1986. Ottawa Charter. Ottawa: WHO; 1986.

[27] Brasil. Lei no 8.080, de 19 de setembro de 1990. Dispõe sobre as condições para a promoção, proteção e recuperação da saúde, a organização e o funcionamento dos serviços correspondentes e dá outras providências. Diário Oficial da União. 1990 set 19.

[28] Brasil. Lei no 11.346, de 15 de setembro de 2006. Cria o Sistema Nacional de Segurança Alimentar e Nutricional (SISAN) com vistas em assegurar o direito humano à alimentação adequada e dá outras providências. Diário Oficial da União. 2006 set 18.

[29] Rawls J. Uma teoria da justiça. São Paulo: Martins Fontes; 1997.

[30] Harvey D. A brief history of neoliberalismo. Oxford University Press: Inglaterra; 2005. Tradução Editora Loyola: São Paulo; 2008.

[31] Friedmann H, McMichael P. Agriculture and the state system: rise and decline of national agricultures, 1870 to the present. Sociologia Ruralis. 1989;XXIX.

[32] Freeman A. Fast Food: Oppression Through Poor Nutrition. Calif. L. Rev. [Internet]. 2007 [acesso em 2020
nov 29];95(6).

https://www.researchgate.net/publication/228246708_F ast_Food_Oppression_Through_Poor_Nutrition/link $/ 5$ 633ca9b08aeb786b7013b76/download

[33] Organización Panamericana de La Salud - OPS. Organización Mundial de La Salud - OMS. Alimentos y bebidas ultraprocesados en América Latina: ventas, fuentes, perfiles de nutrientes e implicaciones. Washington, DC: OPS/OMS; 2019. 